\title{
Design of a Sprout Ultrasonic Aeroponic Cultivation Device
}

\author{
Yi GUO ${ }^{1, a}$, Jiwu MA ${ }^{2, b}$ \\ ${ }^{1}$ Department of Horticulture, Beijing Vocational College of Agriculture, Beijing, 102442, China \\ ${ }^{2}$ Fengtai District Agriculture and Forestry Mechanical Technology Promotion Station, Beijing, \\ 100070, China
}

aguoyibvca@163.com, bhyie1234@sina.com

Keywords: ultrasonic; aeroponic cultivation; sprout

\begin{abstract}
In factory production of sprouts, the layer-rack vertical cultivation devices are usually used and the sprinkling irrigation adopted, which is a severe waste of water resources. Besides, for lack of sunshine, growth of sprouts is hampered. To solve this problem, a sprout ultrasonic aeroponic cultivation device has been designed. Test results show that this device can enable heavy growth of the sprout root system and improve mean yield by more than 15\%, save irrigation water by more than $20 \%$ than the traditional sprinkling irrigation method and can save labor cost by more than 30\% owing to its automatic irrigation. This device features a simple structure, easy operation, safety and reliability, to realize automatic irrigation of sprouts, improve yield of sprouts and save production cost, so it is easy to be promoted in production.
\end{abstract}

\section{Introduction}

Currently, world population is growing continuously and tilth reducing, so the resource issue has become more and more severe. It has become a prominent problem how to improve the utilization ratio of tilth and resources and utilize limited tilth to raise more and more people [1]. The facility agriculture has, to some degree, overcome the time limitation in vegetable production and enhance the utilization ratio of land [2]. Aeroponic cultivation is regarded to be an important facility vegetable cultivation technology and has great prospect. It is a sort of soilless culture, in which case, the root system of vegetables grows in a sealed and light-tight aeroponics space, where oxygen is supplied with the maximum amount to create a good growth conditions for the root field [3]. It features faster growth, more convenient management and fewer man-hours than other cultivation methods [4,5]. Wang Shibin [6] and et al. conducted aeroponic cultivation test on 8 leafy vegetables to conclude that this technology can raise the land utilization rate, increase yield and shorten growing period of these vegetables. Ling Min [7] and et al. found out that aeroponic cultivation was good for growth of plantain, could shorten the growth period, enhance biomass and improve nutritional quality. Gao Jianmin [8] and et al. concluded that aeroponic cultivation could evidently promote biomass accumulation of chia, potherb fameflower and eryngium foetidum. The present aeroponic cultivation mostly adopts the pressure-type spray and mechanical high-speed rotating centrifugal atomization, which features a large fog drop volume and short residence time in the air, so the root system absorption is poor. By contrast, the ultrasonic atomization features fine fog drops and longer floating and movement in the air. Wen Jing [9] and et al. developed the ultrasonic atomization cultivation device to produce the water fog floating in the air for longer than 1min, so that the root system of vegetables can evenly absorb the nutrition and moisture.

Today in China, the traditional agriculture is changing to the ecological agriculture. Along with development of the physical photoelectric technology, the artificial light source has been more and more widely applied in facility agriculture for replacing or compensating the sunlight. Compared with traditional artificial light sources, LED light source is environmentally friendly, has a longer service life and is free of noise. The LED plant growth light can realize the extremely narrow wave band required by plant growth and enhance light utilization rate by more than $90 \%$. Whereas, the traditional plant growth light has the light utilization rate less than $10 \%$. Obviously, LED plant growth light can greatly save energy $[10,11]$. LED has been regarded to be the best light supply 
method in agriculture, the most promise artificial light source in the agriculture and biological fields in the 21st century and has a good development potential [12,13].

Sprouts are fresh and tender, have unique taste and rich nutrition and are free of pollution, so they are more and more popular with consumers and in the market. They have become fashionable vegetables in the market [14]. Presently, the sprout factories usually adopt the layer-rack vertical cultivation devices and the sprinkling irrigation, which features high space utilization ratio but insufficient sunshine, which is bad for sprout growth and a severe waste of water resources [15].

To solve this problem, a sprout ultrasonic aeroponic cultivation device has been designed to enable heavy growth of the sprout root system, increase yield and save irrigation water. This device features a simple structure, easy operation, safety and reliability, to realize automatic irrigation of sprouts and save production cost, so it is easy to be promoted in production.

\section{Overall Structure and Working Process}

The sprout ultrasonic aeroponic cultivation device is composed of the rack, fog box, supplemental lighting system and control system (Fig. 1). It works like this: first, the control system is used to set the automatic irrigation period, which can be used for a long time or is subject to change as required. In the irrigation stage, the control system controls the ultrasonic atomizer to open and produce water fog, which will be delivered along the main pipe as driven by the fog box fan and along the branch pipe to the cultivation tank as driven by the cultivation tank fan, to realize aeroponic cultivation of sprouts. When the end irrigation time is reached, the irrigation will be stopped and wait for the next cycle. When the water level in the water tank is below the limitation position, the control system will open the water inlet magnetic valve so that water will enter the water tank. When the limited water level is reached, signals will be sent to the control system, which will then shut down the magnetic valve to stop water feeding to the water tank. In the supplemental lighting stage, the control system controls the opening of the supplemental lighting lamp for supplemental lighting; otherwise, the control system will control the closing of the supplemental lighting lamp.

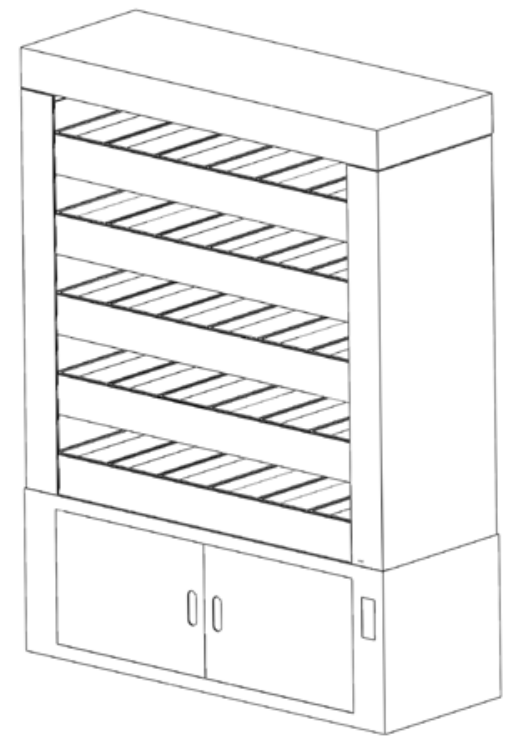

Fig. 1 Overall structure

\section{Structural Design}

Design of the fog box. The fog box is composed of the water tank, filter, magnetic valve, water level sensor, atomizer, fan, flow straightener and spray pipe (Fig. 2). The water tank stores irrigation water. The magnetic valve is connected with the water inlet pipe to control water from the water source to the water tank. The filter purifies water. The water level sensor is designed to detect water flow in the water tank. When the water flow is below the limitation position, the sensor will send signals to the control system, which will open the magnetic valve, when irrigation water will be 
supplied to the water tank. When the limitation water level is reached, the sensor will send signals to the control system to close the magnetic valve, when water supply to the water tank will be stopped. The atomizer is used for atomization treatment of the irrigation water. It adopts ultrasonic atomizer, with parameters shown in Table 1. The atomizer is provided with water level sensor, which will provide protection when the water level is too low. When the water is higher than the limitation water level of the water-level sensor, the spraying will continue. The fan is installed on the water tank and used to enable the atomized irrigation water to spray out of the spray pipe. The flow straightener is installed inside the water tank and guide airflow produced by the fan to act on water fog, which will be delivered along the spray pipe. The spray pipe is composed of the main pipe and the branch pipe, with the former delivery water fog and the latter allocating the water fog to each cultivation tank.

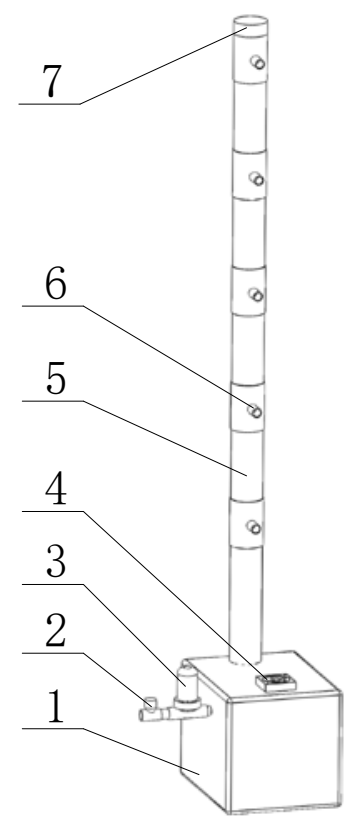

1- water tank 2-magnetic valve 3-filter 4-fan 5-spray main pipe 6-spray branch pipe 7-end plug

Fig. 2 Assembly drawing of the fog box

Table 1 Parameters of the ultrasonic atomizer

\begin{tabular}{c|c}
\hline Items & Parameter \\
\hline Model & SB20*10 \\
\hline Input voltage & AC36V \\
\hline Input current & $6.5-7.0 \mathrm{~A}$ \\
\hline Physical dimension & $254 \times 88 \times 28 \mathrm{~mm}$ \\
\hline Quantity of atomizer plates & 10 \\
\hline Maximum evaporation capacity & $3000 \mathrm{ml} / \mathrm{h}$ \\
\hline Operating environmental temperature & $1-45^{\circ} \mathrm{C}$ \\
\hline Noise & Below $36 \mathrm{~dB}$ \\
\hline
\end{tabular}

Design of the cultivation tank. The seedling tray adopts the special seedling tray for sprouts, made of PP and with dimension $560 \mathrm{~mm} * 240 \mathrm{~mm} * 35 \mathrm{~mm}\left(L^{*} W^{*} H\right)$. Its base is a screen-shape structure. In the growing process, the root of the sprouts can grow downward through the sieve pore to accept atomization cultivation. The cultivation tank is a rectangular structure made of PVC materials through adhesive bonding. Its upper opening is used to place the seeding tray. Each cultivation tank can place 6 seedling trays according to the specification of the seeding tray. The specification of the cultivation tank is $15000 \mathrm{~mm} * 600 \mathrm{~mm} * 150 \mathrm{~mm}\left(L^{*} W^{*} H\right)$ (Fig. 3). On the left side of the cultivation tank is a rectangular opening for installing fans and on the right side a round opening for installing the spray branch pipe. When the device is working, under the action of the fan, water fog flows to the cultivation tank from the spray branch pipe along the fan direction, to fill the entire inside space of the cultivation tank, thereby to ensure evenness of aeroponics and make aeroponic cultivation on sprouts. In the atomization process, some water fog will condense to water, 
which will flow along the spray branch pipe to the spray main pipe and then to the water tank since the cultivation tank base is designed with a $5^{\circ}$ inclination to prevent the settlement of water to the cultivation tank base.

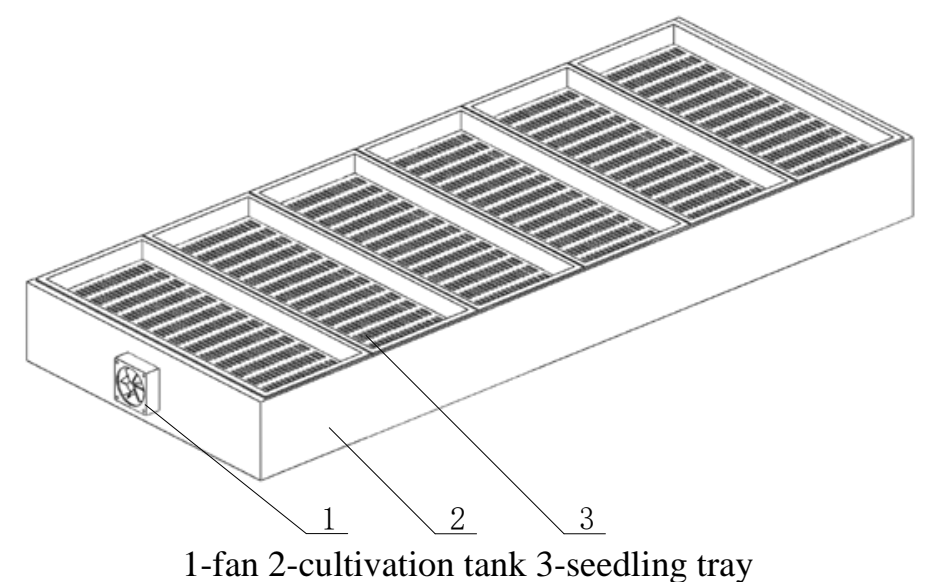

Fig. 3 Assembly drawing of the cultivation tank

Design of the Supplemental Lighting System. To improve space utilization ratio, this device has adopted the layer-rack cultivation structure. In this structure, the layer tack can shade natural lighting, which will effect growth of sprouts. To ensure sufficient sunshine for sprouts cultivated by this device, a supplemental lighting system has been designed. Each layer is provided with 5 light straps with interval of $11 \mathrm{~cm}$ between every two. The light strap is the LED plant growth light, which is composed of the red light bead and the blue light bead, with the wavelength of $650-660 \mathrm{~nm}$ and 460-470nm, respectively and ratio between them 1:1.

Design of the rack. The rack is composed of the base, side frame and top tray. The base is a cabinet structure provided with the French doors. Inside the base, one side is used for placing the fog box and the other for forcing of sprouting. The side frame is welded with the angle steel and steel plate for supporting the cultivation tank. The top tray is used for installing the supplemental lighting lamp for the cultivation tank on it.

Design of the control system. The control system is installed in the base for controlling the fog box fan, cultivation tank fan, supplemental lighting lamp and magnetic valve. It is composed of the main control chip, display, electric relay and DC power supply. The main control chip adopts the 8051 SCM, accompanied with the clock module and electric relay module. There are two electric relays, one for controlling the atomizer, fox box fan and cultivation tank fan, and the other for controlling the supplemental lighting lamp. There are two DC power supplies, one being DC $24 \mathrm{~V}$ for providing power for the atomizer and the other being DC $12 \mathrm{~V}$ for providing power for the supplemental lighting lamp and the fan.

\section{Conclusion}

The sprout ultrasonic aeroponic cultivation device was tested in Beijing Green Valley Sprouts Co, Ltd. Test results show that this device can enable heavy growth of the sprout root system and improve mean yield by more than $15 \%$, save irrigation water by more than $20 \%$ than the traditional sprinkling irrigation method and can save labor cost by more than $30 \%$ owing to its automatic irrigation.

This device features a simple structure, easy operation, safety and reliability, to realize automatic irrigation of sprouts, improve yield of sprouts and save production cost, so it is easy to be promoted in production.

\section{Acknowledgements}

This paper is funded by "Water-saving Irrigation Technology in the Production of Sprouts" project (Project No. 20150203-13) under Beijing Municipal Commission of Rural Affairs. 


\section{References}

[1] Yang Qichang. Developmental strategy of plant factory [J]. Science \& Technology Review. 2014:32(10): 20-24.

[2] Fu Shaoming, Teng Guanghui, Fan Geng, Li Wujie. Effect of Different Cultivation Angle on Lettuce Under Stereoscopic Aeroponic Cultivation [J]. Agricultural Engineering. 2015(S1):39-42.

[3] Guo Yi, Chen Lanfen, Zhang Guiqin. Design of a Sprout Layer-rack Aeroponic Cultivation Device[J]. Advances in Engineering Research. 2016(104):395-400.

[4] Gao Jianmin, Huang Guizhen, Yin Wenchu, Lu Daipeng, Li Junyi, Liu Changjian. Variation of root-zone droplet deposition, temperature and humidity of truss ultrasonic atomization cultivator [J]. Transactions of the Chinese Society of Agricultural Engineering. 2013(6): 185-192.

[5] Liu Yifei, Cheng Ruifeng, Yang Qichang. Aeroponics Control System for Tomato Cultivation in Greenhouse Based on LabVIEW [J]. Journal of Agricultural Mechanization Research. 2015(01): 90-95.

[6] Wang Shibin, Li Baohai, Zhu Rongjie, Yang Bin. Study on Growing Development of Eight Leaf Vegetables with Aeroponical Culture in Greenhouse [J]. Southwest China Journal of Agricultural Sciences. 2015,(04):1854-1856.

[7] Ling Min, He Chunmei, Gao Jianmin, Wang Hongfeng. Effects of Aeroponics on the Growth, Yield and Quality of Plantago asiatica [J]. Forestry and Environmental Science. 2015 (01):29-33.

[8] Gao Jianmin, Ling Min, He Chunmei, Wang Hongfeng. Study on the Three Kinds of Medicinal and Edible Plants Growth in Aeroponics [J]. Forestry and Environmental Science. 2015(01):34-37.

[9] Wen Jing, Cheng Ruifeng, Meng Lili, Wei Jinhe, Yang Qichang, Zhang Jun. Development and Application Effect of Ultrasonic Aeroponic Cultivation Device [J]. Acta Agriculturae Jiangxi. 2012(01):23-25.

[10] Jiang Tian, Zhang Xiao. Intelligent LED System for Plant Growth [J]. China Illuminating Engineering Journal. 2013(S1): 168-172.

[11] Luo Sihan, Chen Ting, Xia Zixiang, Peng Wen, Wang Baijuan. A Design for Intelligent Lighting Control System of Plants Based on LED Light Source[J]. The Food Industry. 2016(02):193-196.

[12] Ye Pusheng, Zhong Binghan, Mo Xingbo, Liang Qidong, Hu Xiaochao, Shi Zhiwei. Fitting Algorithm of LED Plant Growth Spectrum [J]. China Illuminating Engineering Journal. 2015(04): 111-114.

[13] Zhang Yanli, Niu Guoling, Jiang Yongcheng, Niu Jinhua, Bian Ze. Design of a LED Plant Supplemental Lighting Control System Based on SCM[J]. Journal of Anhui Agricultural Sciences. 2016(23): 248-250.

[14] Guo Yi, Zhang Guiqin. Development of A Design System for the Sprout Vertical Cultivation Device[J]. Advances in Engineering Research. 2015(26):206-211.

[15] Guo Yi, Chen Lanfen, Suo Lang Jin Zong, Yang Zhen. Design of Sprout Aeroponics Equipment [J]. Advances in Engineering Research. 2016(88):1783-1789. 